\title{
Cryopreservation of female germplasm in pigs
}

\author{
Tamás Somfai ${ }^{1}$, Kazuhiro Kikuchi², Hiroyuki Kaneko², Junko Noguchi², \\ Koji Yoshioka ${ }^{3}$, Takashi Nagai ${ }^{1}$ \\ ${ }^{1}$ NARO Institute of Livestock and Grassland Science, Tsukuba, Ibaraki 305-0901, Japan; ${ }^{2}$ National \\ Institute of Agrobiological Sciences, Tsukuba, Ibaraki 305-8602, Japan; ${ }^{3}$ NARO Institute of Animal \\ Health, Tsukuba, Ibaraki, Japan
}

\begin{abstract}
Cryopreservation of female germplasm has basic importance in preservation and distribution of genetic lines in farm animals. Although vitrification technology has been applied for the cryopreservation of porcine oocytes and ovarian tissues, reduced developmental competence of preserved oocytes and the lack of offspring produced from them underlines the importance for further developments in cryopreservation protocols for this purpose. This review discusses the problems of female germplasm cryopreservation in pigs and the possible strategies to overcome them and gives an update on the present status of cryopreservation of porcine oocytes and ovarian tissues.
\end{abstract}

\section{Introduction}

Cryopreservation of female germplasm has basic importance in preservation and distribution of genetic lines in farm animals. Recent revival of some indigenous pig breeds in the pork market underlines the importance of the preservation of genetic diversity in pigs (Ratky et al. 2007; Daza et al. 2008). For decades, development of cryopreservation methods of porcine embryos and oocytes was far behind those of other domestic species. This was caused by the high sensitivity of porcine embryos and oocytes to low temperatures, the delayed establishment of in vitro embryo production (IVP) systems and the low quality of resultant oocytes and embryos. Although several research groups have established cryopreservation protocols for porcine oocytes in the last decades, embryo development remained low and there has been no report on piglets produced from cryopreserved oocytes to date. Therefore, oocyte cryopreservation in pigs still represents a challenge to be solved. In the meantime, new technologies for the cryopreservation of ovarian tissues are emerging which provide future possibilities for the cryopreservation of female germline. In the chapters below, we will discuss the problems of the germline cryopreservation in pigs and possible strategies to overcome them and give an update on the present status of cryopreservation of porcine oocytes and ovarian tissues.

\section{Cryopreservation-related damages in porcine oocytes and embryos}

The unique characteristics of porcine oocytes and embryos fundamentally determine their sensitivity to low temperatures and their feasibility to cryopreservation techniques. Porcine oocytes contain 156 ng lipid (McEvoy et al. 2000), which is extremely high, even compared to those of cows (89 ng/oocyte) (Ferguson \& Leese, 1999). In porcine oocytes and embryonic cells, lipid droplets are usually form complexes with cytoskeletal elements, membranes and cytoplasmic organelles and play an important role in metabolism as energy source during 
maturation, fertilization and early embryonic development (Sturmey et al. 2006; Sturmey et al. 2008). When oocytes and embryo cells with high lipid content (such as those of pigs) are cooled below a critical temperature of $15{ }^{\circ} \mathrm{C}$, phase separation of the membrane lipids occurs, causing irreversible damage in membrane structure (Edidin \& Petit 1977; Didion et al. 1990; Gerelchimeg et al. 2009). This phenomenon is often referred as "chilling injury". Furthermore, in those cells which maintain membrane integrity after cooling, sub-lethal damages such as disorganization of the functional units of lipid, mitochondria and endoplasmic reticulum (ER), and altered functions of these cytoplasmic organelles may further reduce their viability and competence (Gerelchimeg et al. 2009). A good example for altered organelle function is the cooling-related release of $\mathrm{Ca}^{2+}$ in the cytoplasm of porcine oocytes from intracellular stores triggering apoptotic cell death (Mattioli et al. 2003). Also, meiotic spindles and cytoskeletal components such as microtubules and microfilaments - which play important roles in distribution and anchoring of cytoskeletal organelles - suffer extensive damage during cooling (Liu et al. 2003a; Yang et al. 2010). Low temperature at the immature stage greatly impairs the cytoplasmic maturation of porcine oocytes during in vitro maturation (IVM) (Liu et al. 2003b). Similarly to cooling, cytoskeleton and mitochondria suffer primal damages during cryopreservation of porcine embryos and oocytes (Dobrinsky et al. 2000; Rojas et al. 2004; Wu et al. 2006; Shi et al. 2007). Accordingly, cytoplasmic functions which require mitochondrial contributions such as regulation of reactive oxygen species (ROS) and calcium levels in cytosol are also compromised in cryopreserved oocytes (Somfai et al. 2007; Gupta et al. 2009; Kim et al. 2011). As a result, apoptosis is frequently observed in cryopreserved porcine embryos and oocytes (Fabian et al. 2005; Cuello et al. 2007a; Vallorani et al. 2012). These examples demonstrate the complexity and interactions among different types of cryopreservation-induced alterations in cryopreserved cells.

\section{Embryo cryopreservation - lessons for oocyte cryopreservation}

In pigs, as in any other mammalian species, development of cryopreservation technologies for embryos was (at least) a step ahead of that of oocytes. The experiences of embryo cryopreservation provide important knowledge for the development of cryopreservation protocols for female germplasm.

Soon after the development of embryo freezing methods it became clear that compared with other farm animals, high susceptibility to chilling injury makes porcine embryos very difficult to preserve by the traditional slow-freezing methods (Wilmut 1972; Polge et al. 1974). Nevertheless, to some extent, traditional slow-freezing could be successfully applied for in vivo produced embryos (Hayashi et al. 1989; Kashiwazaki et al. 1991; Fujino et al. 2007). A major breakthrough in porcine embryo cryopreservation was achieved by Dr. Hiroshi Nagashima and colleagues, who - realizing the contribution of high lipid content to low cryo-tolerance improved chilling tolerance of embryos by removing intracellular lipid droplets mechanically from cleavage stage embryos after polarization of lipids by centrifugation (a technique often referred as "delipation" or "delipidation") (Nagashima et al. 1994). This method combined with slow freezing has led to the production of piglets from cryopreserved porcine embryos for the first time (Nagashima et al. 1995).

As conventional slow freezing of porcine embryos gained limited success, more expectations were placed on the applications of vitrification techniques for embryo cryopreservation in pigs. Vitrification prevents ice crystal formation (Rall \& Fahy 1985) and its rapid cooling and warming rates of vitrification were expected to overcome the problems of membrane injuries caused by chilling (Dobrinsky 2001). Since the early 1990's, different vitrification techniques 
and carriers have been applied on porcine embryos including "open pulled straw" (OPS) (Vajta et al. 1997), "solid surface" (SSV) (Dinnyes et al. 2003), "Cryotop" (= minimum volume cooling, MVC) (Esaki et al. 2004) and "Cryoloop" (Kawakami et al. 2008). As permeable cryoprotectants (CPA), ethylene glycol has been most frequently used alone or combined with dimethyl sulfoxide (DMSO) with approximately similar efficacies when the total CPA concentration was 32-40\% (v/v) (Dinnyes et al. 2003; Cuello et al. 2008). The first piglets from vitrified embryos have been obtained from in vivo derived blastocysts (Dobrinsky et al. 2000; Berthelot et al. 2000; Beebe et al. 2002). Cryopreservation of in vitro produced porcine embryos was a step behind that of their in vivo derived counterparts because of the relatively late development of reliable in vitro fertilization (IVF) and embryo culture systems and the compromised competence of their derivative embryos in this species. Porcine embryos produced by IVF and embryo culture are considered to have lower ability to survive cryopreservation than in vivo produced embryos because the high incidences of polyspermy and the stresses during culture reduce their competence (IVP) embryos (Nagai et al. 2006). In accordance, IVF embryos transferred at the 1-cell stage show higher pregnancy rates compared with those transferred at 2-4 cell after culture (Kikuchi et al. 1999). Suggesting that vitrification and transfer of in vitro produced (IVP) embryos at the early stages may be beneficial by avoiding stresses caused by the culture system we have applied the SSV technique for IVP porcine zygotes which resulted in high survival rates (over 93.4\%), 60\% pregnancy after transfer and the production of piglets from IVP porcine embryos without lipid removal for the first time (Somfai et al., 2009). After IVF, oocytes were centrifuged - which itself does not affect the competence and cryo-tolerance of zygotes (Somfai et al. 2008) - to make the cytoplasm transparent and zygotes were selected for vitrification according to the presence and number of pronuclei omitting highly polyspermic oocytes.

Several strategies have been applied to modify embryo characteristics in order to enhance survival of embryos during vitrification. Dobrinsky et al. (2000) improved survival of porcine expanded blastocyst embryos by depolimerizing microfilaments with cytochalasin B (CB) prior to vitrification. Such treatment is ought to make cell membranes elastic and therefore less susceptible to vitrification-related damages. Combination of delipation at early stages with vitrification for blastocysts also improved survival rates (Ushijima et al. 2004; Esaki et al. 2004) and litter sizes after transfer (Beebe et al. 2005). The first piglets obtained from cryopreserved fertilization-derived IVP porcine embryos were also achieved by delipation of ICSI-derived embryos at the early stages followed by vitrification at the blastocysts stage (Nagashima et al. 2007). On the other hand, mechanical delipation by micromanipulation raises safety concerns since this technique compromises the integrity of zona pellucida clearing the way to pathogen transmissions. To address this point, Esaki et al. (2004) have developed a mechanical delipidation protocol without micromanipulation. Another approach was reported by Men et al. (2006) who improved cryotolerance of IVP blastocysts by chemical enhancement of lipolysis using Forskolin. A fundamentally novel innovation to improve cryotolerance of porcine embryos was introduced by Pribenszky et al. (reviewed in Pribenszky et al. 2010) who induced stress resistance of embryos by applying a non-lethal stress (such as with high hydrostatic pressure) to oocytes. This technology was used for oocytes before nuclear transfer and improved the cryo-tolerance of resultant embryos (Du et al. 2008a) presumably by inducing the production of stress-proteins. These experiences on embryo cryopreservation basically determined the pathway for the establishment of cryopreservation systems for oocytes and several techniques which led to success with embryos have been tested for oocytes in order to improve cryopreservation efficacies.

Regarding embryo vitrification, recent results have demonstrated that vitrification of in vivo-derived porcine blastocysts does not require pretreatment with cytochalasin B and/or 
centrifugation (Cuello et al. 2010). Current vitrification protocols can provide excellent results with in vivo-derived blastocysts without pretreatments, which are time consuming and might be detrimental for embryo quality. Accordingly, in vitro embryo survival similar to those of fresh embryos was reported after vitrification of porcine blastocysts by the superfine OPS (SOPS) method without pretreatments (Sanchez-Osotio et al. 2010).

\section{Cryopreservation of porcine oocytes - why so difficult?}

Similarly to embryos, the large intracellular lipid content in porcine oocytes can be accused for their low cooling tolerance (Ogawa et al., 2010). Nevertheless, mammalian oocytes are generally considered to be more difficult to be cryopreserved compared with embryos (Vajta 2000). Indeed, early works have demonstrated membrane damage in almost all of porcine oocytes subjected to slow freezing (Didion et al. 1990). Although a significant proportion of oocytes can survive vitrification, their developmental competence is always compromised (reviewed by Zhou \& Li, 2009). To date, there is no report on the production of live piglets from cryopreserved oocytes. Compared with multiple-cell embryos, a single-cell oocyte has the obvious problem of a limited ability to recover from vitrification-induced damages and develop into normal embryos - especially, when the IVF/embryo culture system used is stricken with high polyspermy rates and culture stresses, such as in case of pigs. Also, the large size of mammalian oocytes has frequently been accused for making their cryopreservation difficult compared with embryos and other cell types. Nevertheless, high survival rates for zygotes and low survival for oocytes vitrified by the same system dispute if large size of oocytes would be the primarily limiting factor for cryopreservation (Somfai et al. 2008b; Somfai et al. 2012a). Furthermore, despite of their similar size, immature and in vitro matured porcine oocytes show different tolerance for cryopreservation; survival rates after vitrification gradually increase during oocyte maturation and fertilization (Rojas et al. 2004; Somfai et al. 2012a). Ultra-structural changes during oocyte maturation and fertilization are most likely to cause these differences. In pigs, maturation and fertilization is associated with a decrease of lipid content in oocytes (Sturmey \& Leese 2003, Romek et al. 2011) and the translocation of organelles such as mitochondria and ER (Cran 1985; Sun et al. 2002). Also, changes in membrane structure during nuclear progression from the germinal vesicle (GV) stage to the metaphase-II and pronuclear stages in mammalian oocytes are suggested to affect cryo-tolerance by decreasing phase-transition temperatures (Ghetler et al. 2005) and increasing permeability of oocyte membranes to cryoprotective agents (CPAs) (Le Gal et al. 1994; Agca et al. 1998). These suggest that the optimimum protocol for the cryopreservation of porcine oocytes (i.e. cooling and warming rates and CPA treatment) may be dependent on the actual maturation stage.

\section{Cryopreservation of matured oocytes}

Although vitrification was first applied on porcine oocytes at the immature stage (Isachenko et al. 1998), the most effort to preserve porcine oocytes was put in those matured in vitro so far (Zhou \& Li 2009). To date, several vitrification methods have been applied for the cryopreservation of in vitro matured porcine oocytes such as OPS (Rojas et al. 2004, Shi et al. 2006), Cryotop (Fujihira et al. 2004; Ogawa et al. 2010, Galeati et al. 2011) or SSV (Somfai et al. 2006; Gupta et al. 2007). All of these methods have provided surviving rates over 70 $\%$; however, developmental competence is always compromised compared with non-vitrified counterparts. The number of comparative analysis between these methods in porcine oocytes is limited; some results suggest the superiority of Cryotop device compared with OPS method (Liu et al. 2008). To date, the highest survival and developmental rates were achieved by the 
Cryotop method combined with lipid-removal and microtubule stabilization (Ogawa et al. 2010). Vitrification protocols can differ from each other in CPA treatment and vitrification carrier. Among penetrating CPAs, dimethyl sulfoxide (DMSO) and ethylene glycol (EG) have been often used for the vitrification of metaphase-II stage oocytes. Comparing their effects on oocytes, similar survival rates of porcine oocytes after vitrification were reported using these two CPAs (Gupta et al. 2007); however, EG was found to be less toxic than DMSO and therefore appeared more feasible for the purpose (Gupta et al. 2007; Taniguchi et al. 2011). Also, our results have confirmed that treatment of porcine oocytes with EG-based vitrification solution does not affect the developmental ability of oocytes (Somfai et al. 2006). On the other hand, a treatment with a combination of EG with DMSO seems to exert some detrimental effects on matured oocytes (Shi et al. 2006, Shi et al. 2007). Regarding the vitrification carriers, Cryotop and Cryoloop have been found to provide the highest cooling/warming rates in solutions with minimum CPA volume (Sansinena et al. 2011) and therefore may result in high survival rates for porcine oocytes as well. On the other hand, the small capacity (a maximum of 5-10 oocytes per carrier) of these methods (i.e. the number of oocytes preserved at one setting) greatly limits their feasibility for the preservation of porcine oocytes especially considering the relatively low efficacy of IVF or intracytoplasmic sperm injection (ICSI)/embryo culture techniques in pigs. Regarding this problem we have applied a SSV protocol using an EG-based CPA solution as a basic method for the preservation of porcine oocytes (Somfai et al. 2007) modified from the original protocols of Dinnyes et al (2000) and Gupta et al., (2007). This approach allows the vitrification of up to 100 oocytes at one setting in approximately 20 minutes (Fig. 1). After

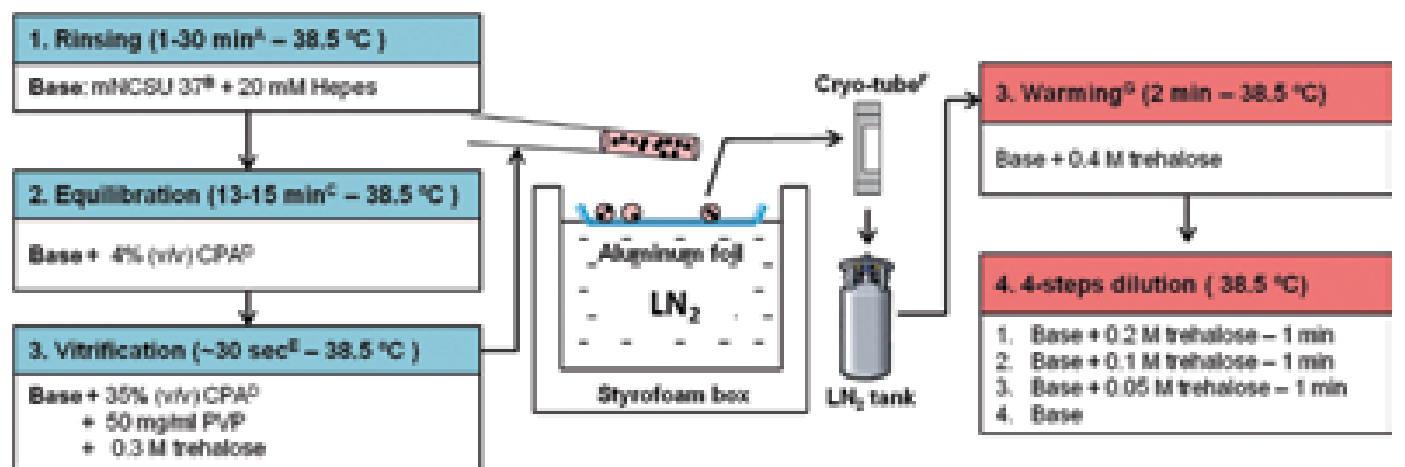

Fig. 1. The schematic diagram of a solid surface vitrification protocol modified for the preservation of porcine oocytes in large quantities.

This protocol under constant improvement.

${ }^{\text {A }}$ Up to 100 oocytes can be processed as one setting. In case of immature oocytes rinsing is performed for 30 min in base medium supplemented with $7.5 \mu \mathrm{g} / \mathrm{mL}$ Cytochalasin B according to Fujihira et al. (2004).

${ }^{B}$ Glucose-free NCSU 37 supplemented with pyruvate and lactate (Kikuchi et al., 2002)

${ }^{\mathrm{C}}$ Up to 100 oocytes can be processed as one setting. In case of immature oocytes rinsing is performed in equilibration medium supplemented with $7.5 \mu \mathrm{g} / \mathrm{mL}$ Cytochalasin B.

D Permeable cryoprotectant (CPA) can be either ethylene glycol or the combination of ethylene glycol and propylene glycol.

E During vitrification, groups of 30-50 oocytes are washed 3 times in $20 \mu$ l of vitrification solution then dropped on the sold surface in 2-3 microdrops ( $\sim 2 \mu$ l each, containing 10-25 oocytes).

F Microdrops prepared at one setting are placed together in cryo-tubes with a pre-cooled forceps.

${ }^{\mathrm{G}}$ Microdrops prepared at one setting are placed together in $2.5 \mathrm{ml}$ warming solution with a pre-cooled forceps.

$\mathrm{LN}_{2}=$ liquid nitrogen 
warming of vitrified oocytes, live ones can be selected either by morphological observation under a stereo microscope or by staining with fluorescein diacetate which stains live oocytes green under UV light without any harmful effect on their competence (Shi et al., 2006).

Despite of the reasonable survival rates for porcine oocytes vitrified at the mature stage, all papers reported their reduced ability to undergo cleavage and to develop to the blastocyst stage and reduced quality of blastocysts in terms of cell numbers (Zhou \& Li 2009). Previous reports have revealed that, during vitrification, matured porcine oocytes suffer various types of cryoinjuries such as the disruption of cytoskeleton (Rojas et al. 2004; Shi et al. 2007; Galeati et al. 2011) and the meiotic spindle (Rojas et al. 2004; Wu et al. 2006; Shi et et al. 2007), mitochondrial damage (Shi et al. 2007) or the premature release of cortical granules (MarcoJimenez et al. 2012). As a result, vitrification is associated with the compromised ability of oocytes to neutralize the intracellular accumulation of reactive oxygen species (Somfai et al. 2007; Gupta et al. 2010), parthenogenetic activation of oocytes (Somfai et al. 2007), reduced penetration rates after IVF (Somfai et al. 2007; Galeati et al. 2011) and the failure of male pronuclear formation after penetration (Somfai et al. 2007). Several strategies have been developed so far to reduce the detrimental effects of vitrification on porcine metaphase-Il oocytes. Mechanical delipation before vitrification significantly improved the developmental competence of cryopreserved oocytes providing excellent developmental rates after parthenogenetic activation (Ogawa et al. 2010); however, this approach has not yet been tested for IVF. Chemical delipation by forskolin treatment during IVM has been reported to result in some improvement on the cryotolerance of porcine oocytes (Fu et al. 2011) although this treatment exerts negative side effects on oocyte competence (Fu et al. 2011; Prates et al. 2012). Unlike in embryos, treatment with CB before vitrification of metaphase-Il stage oocytes does not seem to improve survival rates (Somfai et al. 2006; Gupta et al. 2007; Marco-Jimenez et al. 2012). On the other hand, several studies have confirmed the positive effect of microtubule stabilization by taxol on the competence of vitrified metaphase-Il stage oocytes (Fujihira et al. 2005; Shi et al. 2006; Fu et al. 2009; Ogawa et al. 2010). Since microtubules play an important role in the distribution and anchoring of mitochondria in porcine oocytes, Taxol treatment is also advantageous for the preservation of mitochondrial compartment in vitrified oocytes (Fu et al. 2009). Induced stress resistance either by high hydrostatic pressure or osmotic stress treatment has been reported to improve the postvitrification developmental ability of porcine metaphase-II stage oocytes after parthenogenetic activation (Pribenszky et al. 2008; Du et al. 2008b; Lin et al., 2009); however, this approach has not yet been tested with IVF or ICSI.

\section{Cryopreservation of immature oocytes}

Cryopreservation of oocytes at the immature stages may be inevitable when germ cell preservation for gene banking must be performed under conditions without being able to perform IVM. Also, oocyte cryopreservation at the immature stages, before the formation of meiotic spindle can be advantageous to circumvent the spindle damages and resultant digyny caused by the failure of second polar body extrusion after cryopreservation at the metaphase-II stage (Eroglu et al. 1998). Our results have revealed that, despite of lower survival rates compared with in vitro matured counterparts, porcine cumulus-enclosed oocytes vitrified at the immature GV stage can maintain/restore competence during IVM to undergo maturation and fertilization in a normal manner and the resultant blastocysts appear similar to those obtained from nonvitrified oocytes in terms of cell numbers (Somfai et al. 2010). The obvious disadvantage for the vitrification of immature oocytes seems to be their limited survival rate. A plausible reason for this problem may be the low permeability of oocytes to CPA at the immature stage which has been verified in cattle and goats. This suggestion is supported by our recent results; replacement 
of EG with the more permeable propylene glycol (Pedro et al. 2005) dramatically improved survival rates after vitrification of immature porcine oocytes from $25 \%$ to over $70 \%$ (Somfai et al. 2012b, Fig. 2). Nevertheless, despite of the high survival and normal maturation rates, oocytes vitrified in PG showed very low embryo development due to the toxic side effects of PG (Somfai et al. 2012b). To reduce the toxic effects of PG and to increase survival rates of EG, the combination of EG with PG was proven to be effective, resulting in approximately $40 \%$ survival after vitrification and $10 \%$ blastocyst development after IVF of the surviving oocytes. These results demonstrate that the nature of permeating CPAs fundamentally determines the success of oocyte vitrification and that the optimum CPA treatment may depend on the maturation stage. Survival rates and the competence after vitrification of immature oocytes is affected by several other factors as well. Interestingly, the survival rate of immature oocytes after vitrification seems to be primarily affected by the actual developmental competence of the oocyte population. Our investigations have revealed a strong positive correlation between vitrification-survival at the GV stage and the blastocyst development ratio of non-vitrified oocytes of the same batch (Fig. 3). With other words, survival rate after vitrification reflects the actual developmental competence of an oocyte batch. Similar to these results, the batch-dependence of survival after slow-freezing has also been reported in porcine embryos (Fujino et al. 2007). The timing of vitrification during the rather long period of the GV stage affects the competence of oocytes as well. Our results have revealed that oocytes vitrified after $20 \mathrm{~h}$ culture with the meiosis-inhibitor dbcAMP lose their ability to proceed properly to the MII stage during IVM (Somfai et al. 2012a). In this respect, pre-maturation before vitrification is not recommended.

\section{Treatment - permeable cryoprotectant}

\section{aControl a vitrified - $35 \%$ EG a vitrified $-35 \%$ PG a vitrified $-17.5 \% \mathrm{EG}+17.5 \% \mathrm{PG}$}
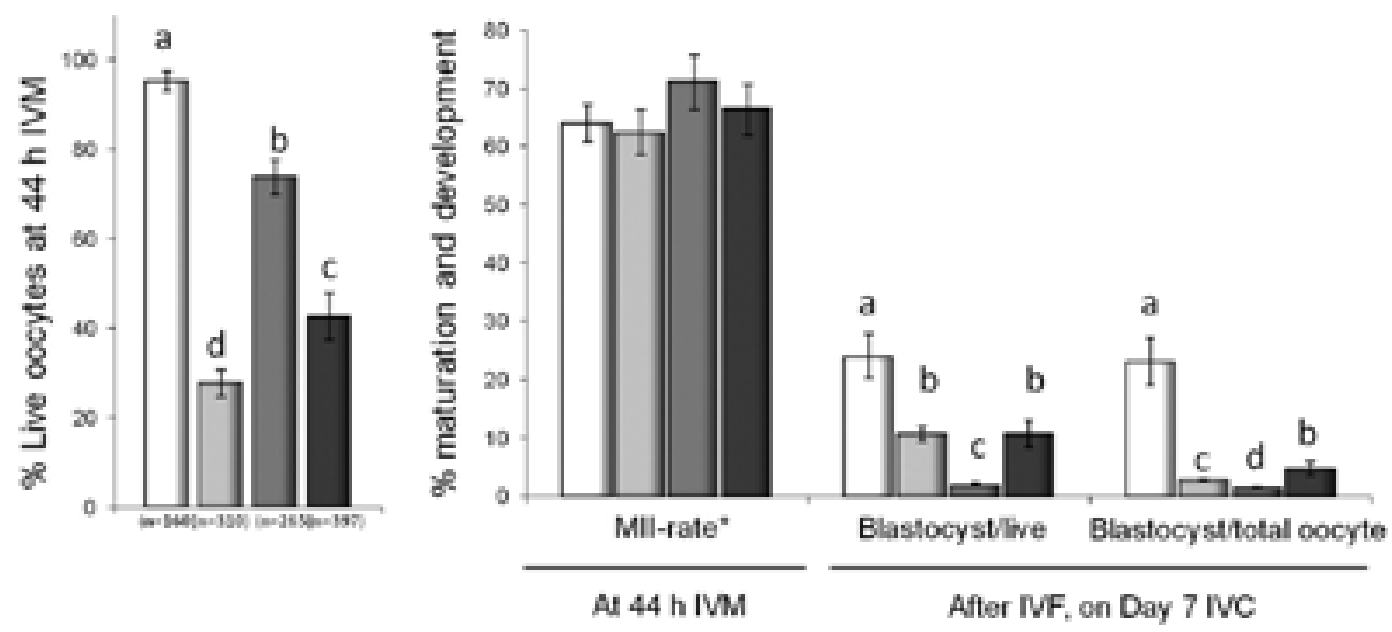

Fig. 2. Survival, maturation and development of immature porcine oocytes vitrified by the SSV method using different CPA combinations.

*MII rate was evaluated by the presence of the first polar body. Oocytes were then subjected to IVF/IVC.

Five replications were performed. Data are presented as mean \pm SEM. Different superscripts denote significant difference at $\mathrm{P}<0.05$.

$\mathrm{EG}=$ ethylene glycol

$\mathrm{PG}=$ propylene glycol

$\mathrm{M}-\mathrm{II}=$ metaphase-II 


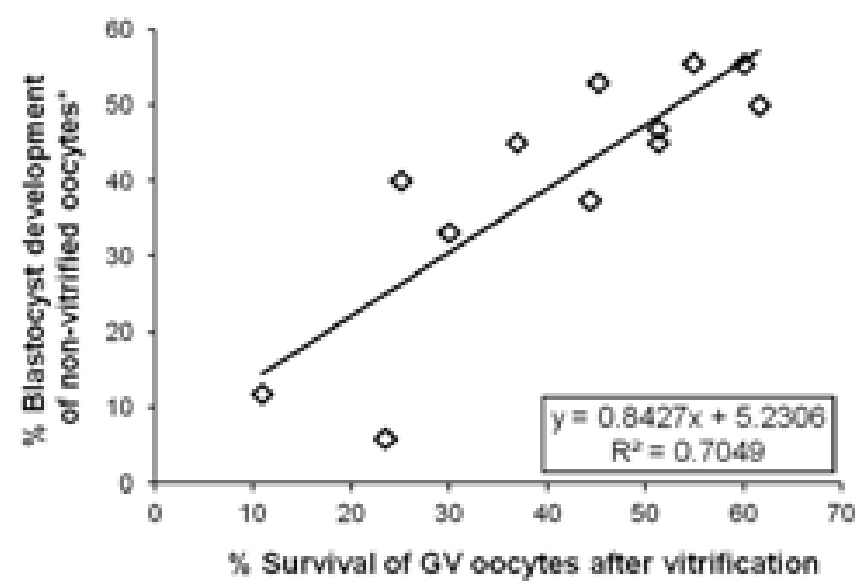

Fig. 3. Correlation between the developmental competence of porcine oocytes and their ability to survive vitrification at the GV stage.

Different batches of oocytes collected on different days were evaluated. Immature cumulusoocytes complexes were either subjected to IVM/IVF/IVC without cryopreservation or vitrified by SSV method in the combination of EG and PG.

* Blastocyst development was calculated from cleaved (thus presumably fertilized) oocytes after IVF using the same batch of oocytes without vitrification.

From the different methods developed to improve cryo-tolerance of porcine embryos delipation has been proven to improve survival rates after vitrification of immature oocytes; however, this may not be an expedient approach since it reduces meiotic competence of oocytes (Park et al. 2005). On the other hand, elastication of the membrane with CB before vitrification - which does not seem to work well for matured oocytes - has been reported to improve survival rates after vitrification of immature porcine oocytes (Ishachenko et al. 1998; Fujihira et al. 2004).

The normal ability of vitrified cumulus enclosed GV-stage oocytes to undergo maturation and fertilization and the high quality of the resultant blastocysts suggest the existence of a recovery mechanism(s) in vitrified immature oocytes during IVM which requires further clarification. Our recent observations indicate that the base medium itself and the optional use of porcine follicular fluid (pFF) as a supplement during IVM greatly affect the ability of vitrified oocytes to undergo nuclear maturation. In NCSU 37 medium defined chemically with polyvinylpyrrolidone, the majority of vitrified GV stage oocytes failed to reach the MII stage whereas reasonable maturation rates were obtained in defined POM medium (Fig. 4) although both media provided over $60 \%$ maturation for non-vitrified oocytes (Yoshioka et al. 2008). Furthermore, the addition of $10 \%$ (v/v) pFF to NCSU 37 medium dramatically increased the maturation ability of vitrified immature oocytes (Fig. 4). Also, preservation of mitochondrial functions during IVM by the inhibition of mitochondrial permeability transition helps vitrified immature porcine oocytes to regain their meiotic competence demonstrating that cytoplasmic recovery can be enhanced during IVM by specific reagents (Nakagawa et al. 2008). Understanding and enhancement of recovery mechanisms during IVM of vitrified immature oocytes may be a potential way to improve developmental rates to the blastocyst stage in the future. Furthermore, the transfer of their nuclear material from oocytes compromised by vitrification into cytoplasts of healthy oocytes offers an alternative way to preserve genetic material of female germline in pigs (Nakagawa et al. 2011). 


\section{Maturation medium \\ $\square \overline{N C S U-37+P V A ~ a N C S U-37+p F F ~ a P O M+P V A ~ a ~ P O M+p F F ~}$}
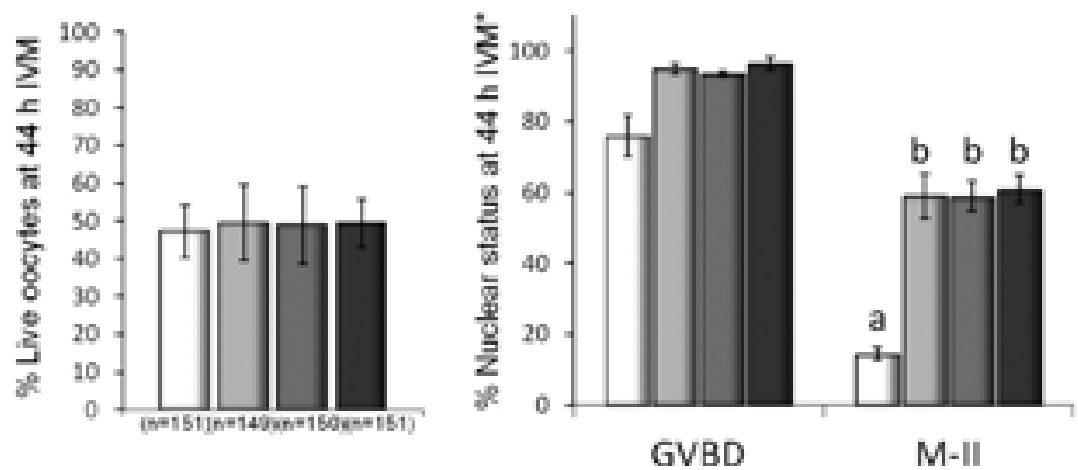

Fig. 4. Survival rates after IVM and nuclear maturation in different media of vitrified immature porcine oocytes.

Porcine oocytes were vitrified by SSV using a combination of EG and PG as permeable CPA. Four replications were performed. Data are presented as mean \pm SEM. Different superscripts denote significant difference at $\mathrm{P}<0.05$.

GVBD = germinal vesicle breakdown

M-II = metaphase-II

\section{Cryopreservation of ovarian tissues}

Cryopreservation of ovarian tissues and whole ovaries is an emerging technology for fertility preservation in humans and gene banking in animals (Johnson \& Patrizio 2011; Santos et al. 2010). The ability of immature oocytes to recover from vitrification-related damages during post-warming culture suggests that the earlier the developmental stage in which the female germplasm is cryopreserved the better chance it may have to regain its developmental competence if proper conditions are provided. This suggestion justifies the idea of preservation of ovarian tissue segments or whole ovaries in pigs. Although there have been some attempts in pigs to preserve ovarian tissues and whole ovaries either by slow-freezing or vitrification, these cryopreserved tissues were not utilized for practical reproduction (reviewed by Santos et al. 2010). Utilization of cryopreserved tissues for reproduction requires the immediate transfer after thawing or warming of cryopreserved samples into recipients or an advanced technology for tissue culture after thawing. However, in pigs, in vitro culture of ovarian tissues and follicles is not in an advanced stage yet and, unlike in mice or cattle, there is no report on live offspring from in vitro grown porcine oocytes to date (Miyano 2005; Hirao 2011). On the other hand, as an alternative to in vitro culture, ovarian tissue segments (both fresh and vitrified) can be transplanted into immune-deficient mice (a technique called "xenografting") which can start folliculogenesis under the proper hormonal environment (Kaneko et al. 2003, 2006; Moniruzzaman et al. 2009). In our laboratory, we have applied a vitrification protocol for the cryopreservation of ovarian tissue segments (Kikuchi et al. 2010). The ovarian tissue from 20 days old piglets was minced into cubes of about 1.0 to $2.0 \mathrm{~mm}$. The vitrification procedure was similar to that described earlier for oocytes with modifications. The tissues were incubated in the vitrification solution employing 35\% EG as permeable cryoprotectant either for $45 \mathrm{~s}$ or $7 \mathrm{~min}$ (45-s and 7-min immersion groups, respectively), then dropped with about 4 
$\mu \mathrm{L}$ of vitrification solution into liquid nitrogen. After storage for several months, micro-droplets containing an ovarian tissue segment were warmed the same way as described for oocytes. Twenty to 30 pieces of tissue were grafted into the kidney capsules of ovariectomized nude mice. When antral follicles were evident in the grafts, oocytes were collected, matured and fertilized in vitro using our standard protocol (Kikuchi et al. 2002). Ovaries containing antral follicles were obtained between 2 to 4 months after grafting from 6 out of 12 mice in both the 45-s and 7-min groups. The collected oocytes were cultured and the maturation rates calculated on the basis of 1 st polar body extrusion were $18 \%$ (7/39) and 33\% (16/49), respectively. After IVF, rates for sperm-penetrated oocytes (83\% and $88 \%$, respectively) and those with normal male and female pronuclei (100\% of penetrated oocytes in both groups) did not differ between in the two immersion groups. These results demonstrate that fully grown porcine oocytes can be collected from primordial follicles that have been cryopreserved and xenografted into nude mice. Despite of their low ability to undergo maturation, those which reach the matured stage during IVM gain the ability to be fertilized by IVF irrespective of the period of immersion. Further research is necessary to optimize vitrification/warming parameters and IVM culture systems to increase maturation rates for oocytes obtained from vitrified ovarian tissue segments.

\section{Summary}

Despite of recent advances in embryo vitrification in pigs, cryopreservation technology for female germplasm requires substantial development for future success. The application of high capacity containers with high cooling/warming rates enabling the effective and quick vitrification of oocytes at large quantities would be advantageous. Also, further works are required to find the optimum CPA treatment and warming procedures which provide the highest survival and embryo development rates. Improvement of culture systems to support post-warming recovery processes in surviving vitrified oocytes may be another potent way to increase efficacy. To do so, further research is necessary to understand the effects of cryopreservation on cellular processes and to characterize recovery mechanisms in oocytes. Emerging technologies, such as cytoplasm transfer may offer alternative ways to reset the developmental competence of oocytes compromised by cryopreservation.

\section{References}

Agca Y, Liu J, Peter AT, Critser ES \& Critser JK 1998 Effect of developmental stage on bovine oocyte plasma membrane water and cryoprotectant permeability characteristics. Molecular Reproduction and Development 49 408-415.

Beebe LF, Cameron RD, Blackshaw AW, Higgins A \& Nottle MB 2002 Piglets born from centrifuged and vitrified early and peri-hatching blastocysts. Theriogenology 57 2155-2165.

Beebe LF, Cameron RD, Blackshaw AW \& Keates HL 2005 Changes to porcine blastocyst vitrification methods and improved litter size after transfer. Theriogenology 64 879-890.

Berthelot F, Martinat-Botte F, Locatelli A, Perreau C \& Terqui M 2000 Piglets born after vitrification of embryos using the open pulled straw method. Cryobiology 41 116-124.

Berthelot F, Martinat-Botte F, Perreau C \& Terqui M 2001 Birth of piglets after OPS vitrification and transfer of compacted morula stage embryos with intact zona pellucida. Reproduction Nutrition and Development 41 267-272.

Cuello C, Berthelot F, Delaleu B, Venturi E, Pastor LM, Vazquez JM, Roca J, Martinat-Botté F \& Martinez EA 2007 The effectiveness of the stereomicroscopic evaluation of embryo quality in vitrified-warmed porcine blastocysts: an ultrastructural and cell death study. Theriogenology 67 970-982.

Cuello C, Sanchez-Osorio J, Almiñana C, Gil MA, Perals ML, Lucas X, Roca J, Vazquez JM \& Martinez EA 2008 Effect of the cryoprotectant concentration on the in vitro embryo development and cell proliferation of OPS-vitrified porcine blastocysts. Cryobiology $\mathbf{5 6}$ 189-194.

Cuello C, Sanchez-Osorio J, Almiñana C, Gil MA, Parrilla I, Roca J, Vazquez JM, Martinez EA \& RodriguezMartinez H 2010 Superfine open pulled straws vitrification of porcine blastocysts does not require 
pretreatment with cytochalasin B and/or centrifugation. Reproduction Fertility and Development 22 808-817.

Cran DG 1985 Qualitative and quantitative structural changes during pig oocyte maturation. Journal of Reproduction and Fertility 74 237-245.

Daza A, Olivares A, Rey AI, Ruiz J \& Lopez-Bote CJ 2008 Iberian pig production: the problems of success. Options Méditerranéennes Série A : Séminaires Méditerranéens 78 163-171.

Didion BA, Pomp D, Martin MJ, Homanics GE \& Markert CL 1990 Observations on the cooling and cryopreservation of pig oocytes at the germinal vesicle stage. Journal of Animal Science 68 2803-2810.

Dinnyes A, Kikuchi K, Watanabe A, Fuchimoto D, Iwamoto M, Kaneko H, Noguchi J, Somfai T, Onishi A \& Nagai T 2003 Successful cryopreservation of in vitroproduced pig embryos by the solid surface vitrification (SSV) method. Theriogenology 59299 (abst)

Dinnyés A, Dai Y, Jiang S \& Yang X 2000 High developmental rates of vitrified bovine oocytes following parthenogenetic activation, in vitro fertilization, and somatic cell nuclear transfer. Biology of Reproduction 63 513-518.

Dobrinsky JR, Pursel VG, Long CR \& Johnson LA 2000 Birth of piglets after transfer of embryos cryopreserved by cytoskeletal stabilization and vitrification. Biology of Reproduction 62 564-570.

Dobrinsky JR 2001 Cryopreservation of swine embryos: a chilly past with a vitrifying future. Theriogenology 56 1333-1344.

Du Y, Lin L, Schmidt M, Bøgh IB, Kragh PM, Sørensen CB, Li J, Purup S, Pribenszky C, Molnár M, Kuwayama M, Zhang X, Yang H, Bolund L \& Vajta G 2008a High hydrostatic pressure treatment of porcine oocytes before handmade cloning improves developmental competence and cryosurvival. Cloning and Stem Cells 10 325-330.

Du Y, Pribenszky CS, Molnár M, Zhang X, Yang H, Kuwayama M, Pedersen AM, Villemoes K, Bolund L \& Vajta G 2008b High hydrostatic pressure: a new way to improve in vitro developmental competence of porcine matured oocytes after vitrification. Reproduction 135 13-17.

Edidin M \& Petit VA 1977 The effect of temperature on the lateral diffusion of the plasma membrane protein. In: Elliott K, Whelan J (eds), The Freezing of Mammalian Embryos. Amsterdam: Elsevier, Excerpta Medica; 155-174.

Eroglu A, Toner M, Leykin L \& Toth TL 1998 Cytoskeleton and polyploidy after maturation and fertilization of cryopreserved germinal vesicle-stage mouse oocytes. Journal of Assisted Reproduction and Genetics 15 447-454.

Esaki R, Ueda H, Kurome $M$, Hirakawa K, Tomii R, Yoshioka H, Ushijima H, Kuwayama M \& Nagashima H 2004 Cryopreservation of porcine embryos derived from in vitro-matured oocytes. Biology of Reproduction 71 432-437.

Fabian D, Gjørret JO, Berthelot F, Martinat-Botté F \& Maddox-Hyttel P 2005 Ultrastructure and cell death of in vivo derived and vitrified porcine blastocysts. Mol ecular Reproduction and Development 70 155-165.

Ferguson EM \& Leese HJ 1999 Triglyceride content of bovine oocytes and early embryos. Journal of Reproduction and Fertility 116 373-378.

Fu XW, Shi WQ, Zhang QJ, Zhao XM, Yan CL, Hou YP, Zhou GB, Fan ZQ, Suo L, Wusiman A, Wang YP \& Zhu SE 2009 Positive effects of Taxol pretreatment on morphology, distribution and ultrastructure of mitochondria and lipid droplets in vitrification of in vitro matured porcine oocytes. Animal Reproduction Science 115 158-168.

Fu XW, Wu GQ, Li JJ, Hou YP, Zhou GB, Lun-Suo, Wang YP \& Zhu SE 2011 Positive effects of Forskolin (stimulator of lipolysis) treatment on cryosurvival of in vitro matured porcine oocytes. Theriogenology 75 268-275.

Fujihira T, Kishida R \& Fukui Y 2004 Developmental capacity of vitrified immature porcine oocytes following ICSI: effects of cytochalasin B and cryoprotectants. Cryobiology 49 286-290.

Fujihira T, Nagai H \& Fukui Y 2005 Relationship between equilibration times and the presence of cumulus cells, and effect of taxol treatment for vitrification of in vitro matured porcine oocytes. Cryobiology 51 339-343.

Fujino Y, Kikuchi K, Nakamura Y, Kobayashi H, Yonemura I, Suzuki M, Misumi K \& Nagai T. 2007 Batchwise assessment of porcine embryos for cryotolerance. Theriogenology 67 413-422.

Galeati G, Spinaci M, Vallorani C, Bucci D, Porcu E \& Tamanini C 2011 Pig oocyte vitrification by cryotop method: effects on viability, spindle and chromosome configuration and in vitro fertilization. Animal Reproduction Science 127 43-49.

Gerelchimeg B, Li-Qing L, Zhong Z, Jiang-Tian T, QingRan K, Jun S, Xue-Dong W \& Zhong-Hua L 2009 Effect of chilling on porcine germinal vesicle stage oocytes at the subcellular level. Cryobiology 59 54-58.

Ghetler Y, Yavin S, Shalgi R \& Arav A 2005 The effect of chilling on membrane lipid phase transition in human oocytes and zygotes. Human Reproduction 20 3385-3389.

Gupta MK, Uhm SJ \& Lee HT 2007 Cryopreservation of immature and in vitro matured porcine oocytes by solid surface vitrification. Theriogenology 67 238-248.

Gupta MK, Uhm SJ \& Lee HT 2010 Effect of vitrification and beta-mercaptoethanol on reactive oxygen species activity and in vitro development of oocytes vitrified before or after in vitro fertilization. Fertility and Sterility 93 2602-2607.

Hayashi S, Kobayashi K, Mizuno J, Saitoh K \& Hirano S 1989 Birth of piglets from frozen embryos. Veterinary Record 125 43-44.

Hirao Y 2011 Conditions affecting growth and developmental competence of mammalian oocytes in vitro. Animal Science Journal 82 187-197.

Isachenko V, Soler C, Isachenko E, Perez-Sanchez F \& Grishchenko V 1998 Vitrification of immature porcine oocytes: effects of lipid droplets, temperature, cytoskeleton, and addition and removal of 
cryoprotectant. Cryobiology 36 250-253.

Johnson J \& Patrizio P 2011 Ovarian cryopreservation strategies and the fine control of ovarian follicle development in vitro. Annals of the New York Academy of Sciences 1221 40-46.

Kaneko H, Kikuchi K, Noguchi J, Hosoe M \& Akita T 2003 Maturation and fertilization of porcine oocytes from primordial follicles by a combination of xenografting and in vitro culture. Biology of Reproduction 69 1488-1493.

Kaneko H, Kikuchi K, Noguchi J, Ozawa M, Ohnuma K, Maedomari N \& Kashiwazaki N 2006 Effects of gonadotropin treatments on meiotic and developmental competence of oocytes in porcine primordial follicles following xenografting to nude mice. Reproduction 131 279-288.

Kashiwazaki N, Ohtani S, Miyamoto K \& Ogawa S 1991 Production of normal piglets from hatched blastocysts frozen at -196 degrees C. Veterinary Record 128 256-257.

Kawakami M, Kato Y \& Tsunoda Y 2008 The effects of time of first cleavage, developmental stage, and delipidation of nuclear-transferred porcine blastocysts on survival following vitrification. Animal Reproduction Science 106 402-411.

Kikuchi K, Kashiwazaki N, Noguchi J, Shimada A, Takahashi R, Hirabayashi M, Shino M, Ueda M \& Kaneko H 1999 Developmental competence, after transfer to recipients, of porcine oocytes matured, fertilized, and cultured in vitro. Biology of Reproduction 60 336-340.

Kikuchi K, Onishi A, Kashiwazaki N, Iwamoto M, Noguchi J, Kaneko H, Akita T \& Nagai T 2002 Successful piglet production after transfer of blastocysts produced by a modified in vitro system. Biology of Reproduction 66 1033-1041.

Kikuchi K, Kashiwazaki N, Nakai N, Noguchi J, Ito J \& Kaneko H 2010 In vitro maturation and fertilization of oocytes from ovarian tissues cryopreserved and xenografted into nude mice. Reproduction Fertility and Development 22 206-207. (abst)

Kim BY, Yoon SY, Cha SK, Kwak KH, Fissore RA, Parys JB, Yoon TK \& Lee DR 2011 Alterations in calcium oscillatory activity in vitrified mouse eggs impact on egg quality and subsequent embryonic development. Pflugers Archive 461 515-526.

Le Gal F, Gasqui P \& Renard JP 1994 Differential osmotic behavior of mammalian oocytes before and after maturation: a quantitative analysis using goat oocytes as a model. Cryobiology 31 154-170.

Lin L, Kragh PM, Purup S, Kuwayama M, Du Y, Zhang X, Yang H, Bolund L, Callesen H \& Vajta G 2009 Osmotic stress induced by sodium chloride, sucrose or trehalose improves cryotolerance and developmental competence of porcine oocytes. Reproduction Fertility and Development 21 338-344.

Liu RH, Sun QY, Li YH, Jiao LH \& Wang WH 2003a Effects of cooling on meiotic spindle structure and chromosome alignment within in vitro matured porcine oocytes. Molecular Reproduction and
Development 65 212-218.

Liu RH, Sun QY, Li YH, Jiao LH \& Wang WH 2003 b Maturation of porcine oocytes after cooling at the germinal vesicle stage. Zygote 11 299-305.

Liu Y, Du Y, Lin L, Li J, Kragh PM, Kuwayama M, Bolund L, Yang H \& Vajta G 2008 Comparison of efficiency of open pulled straw (OPS) and Cryotop vitrification for cryopreservation of in vitro matured pig oocytes. Cryo Letters 29 315-320.

Marco-Jiménez F, Casares-Crespo L \& Vicente JS 2012 Effect of cytochalasin B pre-treatment of in vitro matured porcine oocytes before vitrification. Cryo Letters 33 24-30.

Marco-Jiménez F, Casares-Crespo L \& Vicente JS 2012 Porcine oocyte vitrification in optimized low toxicity solution with open pulled straws. Zygote 29 1-9.

Mattioli M, Barboni B, Gioia L \& Loi P 2003 Cold-induced calcium elevation triggers DNA fragmentation in immature pig oocytes. Molecular Reproduction and Development 65 289-297.

McEvoy TG, Coull GD, Broadbent PJ, Hutchinson JS \& Speake BK 2000 Fatty acid composition of lipids in immature cattle, pig and sheep oocytes with intact zona pellucida. Journal of Reproduction and Fertility 118 163-170.

Men H, Agca Y, Riley LK \& Critser JK 2006 Improved survival of vitrified porcine embryos after partial delipation through chemically stimulated lipolysis and inhibition of apoptosis. Theriogenology 66 2008-2016.

Miyano T 2005 JSAR Outstanding Research Award. In vitro growth of mammalian oocytes. Journal of Reproduction and Development 51 169-176.

Moniruzzaman M, Bao RM, Taketsuru H \& Miyano T 2009 Development of vitrified porcine primordial follicles in xenografts. Theriogenology 72 280-288.

Nagai T, Funahashi H, Yoshioka K \& Kikuchi K 2006 Up date of in vitro production of porcine embryos. Frontiers in Bioscience 11 2565-2573.

Nagashima H, Kashiwazaki N, Ashman RJ, Grupen CG, Seamark RF \& Nottle MB 1994. Removal of cytoplasmic lipid enhances the tolerance of porcine embryos to chilling. Biology of Reproduction 51 618-622.

NagashimaDH, KashiwazakidN, AshmanDRJ, GrupenDCG \& Nottle MB 1995 Cryopreservation of porcine embryos. Nature 374416.

Nagashima H, Hiruma K, Saito H, Tomii R, Ueno S, Nakayama N, Matsunari H \& Kurome M 2007 Production of live piglets following cryopreservation of embryos derived from in vitro-matured oocytes. Biology of Reproduction 76 900-905.

Nakagawa S, Yoneda A, Hayakawa K \& Watanabe T 2008 Improvement in the in vitro maturation rate of porcine oocytes vitrified at the germinal vesicle stage by treatment with a mitochondrial permeability transition inhibitor. Cryobiology 57 269-275.

Nakagawa S, Maedomari N, Kikuchi K, Nagai T, Miyano T, Fulka J Jr \& Manabe N 2011 Vitrification of fully grown and growing porcine oocytes using germinal vesicle transfer. Journal of Reproduction and 
Development 57 335-341.

Ogawa B, Ueno S, Nakayama N, Matsunari H, Nakano K, Fujiwara T, Ikezawa Y \& Nagashima H 2010 Developmental ability of porcine in vitro matured oocytes at the meiosis II stage after vitrification. Journal of Reproduction and Development 56 356-361.

Park KE, Kwon IK, Han MS \& Niwa K 2005 Effects of partial removal of cytoplasmic lipid on survival of vitrified germinal vesicle stage pig oocytes. Journal of Reproduction and Development 51 151-160.

Pedro PB, Yokoyama E, Zhu SE, Yoshida N, Valdez DM Jr, Tanaka M, Edashige K \& Kasai M 2005 Permeability of mouse oocytes and embryos at various developmental stages to five cryoprotectants. Journal of Reproduction and Development 51 235-246.

Polge C, Wilmut I \& Rowson LEA 1974 The low temperature preservation of cow, sheep and pig embryos. Cryobiology 11560

Prates EG, Marques CC, Baptista MC, Vasques MI, Carolino N, Horta AE, Charneca R, Nunes JT \& Pereira RM 2012 Fat area and lipid droplet morphology of porcine oocytes during in vitro maturation with trans-10, cis-12 conjugated linoleic acid and forskolin. Animal 26 1-8.

Pribenszky C, Du Y, Molnár M, Harnos A \& Vajta G 2008 Increased stress tolerance of matured pig oocytes after high hydrostatic pressure treatment. Animal Reproduction Science 106 200-207.

Pribenszky C, Vajta G, Molnar M, Du Y, Lin L, Bolund L \& Yovich J 2010 Stress for stress tolerance? A fundamentally new approach in mammalian embryology. Biology of Reproduction 83 690-697.

Rall WF \& Fahy GM 1985 Ice-free cryopreservation of mouse embryos at -196 degrees $C$ byvitrification. Nature 313 573-575.

Ratky J, Toth P, Egerszegi I, Sarlos P, Manabe N \& Brussow KP 2007 Multifunctional aspects of Mangalica breeding. In:Proceedings of the 5th Vietnamese-Hungarian International Conference on "Animal Production and Aquaculture for Sustainable Farming"; Can Tho, Vietnam. 42-44.

Rojas C, Palomo MJ, Albarracín JL \& Mogas T 2004 Vitrification of immature and in vitro matured pig oocytes: study of distribution of chromosomes, microtubules, and actin microfilaments. Cryobiology 49 211-220.

Romek M, Gajda B, Krzysztofowicz E, Kepczynski M \& Smorag Z 2011 New technique to quantify the lipid composition of lipid droplets in porcine oocytes and pre-implantation embryos using Nile Red fluorescent probe. Theriogenology 75 42-54.

Sanchez-Osorio J, Cuello C, Gil MA, Parrilla I, Maside C, Almiñana C, Lucas X, Roca J, Vazquez JM \& Martinez EA. 2010 Vitrification and warming of in vivo-derived porcine embryos in a chemically defined medium. Theriogenology 73 300-308.

Santos RR, Amorim C, Cecconi S, Fassbender M, Imhof $M$, Lornage J, Paris $M$, Schoenfeldt V \& Martinez-Madrid B 2010 Cryopreservation of ovarian tissue: an emerging technology for female germline preservation of endangered species and breeds. Animal Reproduction Science 122 151-163.

Sansinena M, Santos MV, Zaritzky N \& Chirife J 2011 Numerical simulation of cooling rates in vitrification systems used for oocyte cryopreservation. Cryobiology 63 32-37.

Shi WQ, Zhu SE, Zhang D, Wang WH, Tang GL, Hou YP \& Tian SJ 2006 Improved development by Taxol pretreatment after vitrification of in vitro matured porcine oocytes. Reproduction 131 795-804.

Shi LY, Jin HF, Kim JG, Mohana Kumar B, Balasubramanian S, Choe SY \& Rho GJ 2007 Ultra-structural changes and developmental potential of porcine oocytes following vitrification. Animal Reproduction Science 100 128-140.

Somfai T, Dinnyes A, Sage D, Marosan M, Carnwath JW, Ozawa M, Kikuchi K \& Niemann H 2006 Development to the blastocyst stage of parthenogenetically activated in vitro matured porcine oocytes after solid surface vitrification (SSV). Theriogenology 66 415-422.

Somfai T, Ozawa M, Noguchi J, Kaneko H, Karja NWK, Farhudin M, Dinnyes A, Nagai T \& Kikuchi K 2007 Developmental competence of in vitro-fertilized porcine oocytes after in vitro maturation and solid surface vitrification: Effect of cryopreservation on oocyte antioxidative system and cell cycle stage. Cryobiology 55 115-126.

Somfai T, Kashiwazaki N, Ozawa M, Nakai M, Maedomari N, Noguchi J, Kaneko H, Nagai T \& Kikuchi K 2008 Effect of centrifugation treatment before vitrification on the viability of porcine mature oocytes and zygotes produced in vitro. Journal of Reproduction and Development 54 149-155.

Somfai T, Ozawa M, Noguchi J, Kaneko H, Nakai M, Maedomari N, Ito J, Kashiwazaki N, Nagai T \& Kikuchi K 2009 Live piglets derived from in vitroproduced zygotes vitrified at the pronuclear stage. Biology of Reproduction 80 42-49.

Somfai T, Noguchi J, Kaneko H, Nakai M, Ozawa M, Kashiwazaki N, Egerszegi I, Rátky J, Nagai T \& Kikuchi K 2010 Production of good-quality porcine blastocysts by in vitro fertilization of follicular oocytes vitrified at the germinal vesicle stage. Theriogenology 73 147-156.

Somfai T, Kikuchi K \& Nagai T 2012a Factors affecting cryopreservation of porcine oocytes. Journal of Reproduction and Development 58 17-24.

Somfai T, Kikuchi K, Nakai M, Kaneda M, Akagi S, Watanabe S, Haraguchi S, Geshi M \& Nagai T 2012b A combination of ethylene glycol and propylene glycol is superior to individual cryoprotectants for the vitrification of immature porcine oocytes. Reproduction Fertility and Development 25 187. (abst)

Sturmey RG \& Leese HJ 2003 Energy metabolism in pig oocytes and early embryos. Reproduction 126 197-204.

Sturmey RG, O'Toole PJ \& Leese HJ 2006 Fluorescence resonance energy transfer analysis of mitochondrial:lipid association in the porcine oocyte. Reproduction 132 829-837. 
Sturmey RG, Reis A, Leese HJ \& McEvoy TG 2009 Role of fatty acids in energy provision during oocyte maturation and early embryo development. Reproduction in Domestic Animals 44(Suppl 3) 50-58

Sun QY, Wu GM, Lai L, Park KW, Cabot R, Cheong HT, Day BN, Prather RS \& Schatten H 2002 Translocation of active mitochondria during pig oocyte maturation, fertilization and early embryo development in vitro. Reproduction 122 155-163.

Taniguchi M, Arikawa R, Kaedei Y, Tanihara F, Namula Z, Viet VL, Sato Y \& Otoi T 2011 Effects of cryoprotectant agents and equilibration methods on developmental competence of porcine oocytes. Cryo Letters 32 410-414.

Ushijima H, Yoshioka H, Esaki R, Takahashi K, Kuwayama M, Nakane T \& Nagashima H 2004 Improved survival of vitrified in vivo-derived porcine embryos. Journal of Reproduction and Development 50 481-486.

Vajta G, Holm P, Greve T \& Callesen H 1997 Vitrification of porcine embryos using the open pulled straw (OPS) method. Acta Veterinaria Scandinavia 38 349-352.

Vajta G 2000 Vitrification of the oocytes and embryos of domestic animals. Animal Reproduction Science 60-61 357-364.
Vallorani C, Spinaci M, Bucci D, Porcu E, Tamanini C \& Galeati G 2012 Pig oocyte vitrification by Cryotop method and the activation of the apoptotic cascade. Animal Reproduction Science 135 68-74.

Wilmut I 1972 The low temperature preservation of mammalian embryos. Journal of Reproduction and Fertility 31 513-514.

Wu C, Rui R, Dai J, Zhang C, Ju S, Xie B, Lu X \& Zheng X 2006 Effects of cryopreservation on the developmental competence, ultrastructure and cytoskeletal structure of porcine oocytes. Molecular Reproduction and Development 73 1454-1462.

Yang CR, Miao DQ, Zhang QH, Guo L, Tong JS, Wei Y, Huang X, Hou Y, Schatten H, Liu Z \& Sun QY 2010 Short-term preservation of porcine oocytes in ambient temperature: novel approaches. PLoS One 5 e14242.

Yoshioka K, Suzuki C \& Onishi A 2008 Defined system for in vitro production of porcine embryos using a single basic medium. Journal of Reproduction and Development 54 208-213.

Zhou GB \& Li N 2009 Cryopreservation of porcine oocytes: recent advances. Molecular Human Reproduction 15 279-285. 